\title{
LOS DATOS DE SALUD RELATIVOS A LA INFORMACIÓN SOBRE DOPAJE DE UN DEPORTISTA: LA AUDIENCIA NACIONAL SE PRONUNCIA SOBRE SU ESPECIAL PROTECCIÓN*
}

\author{
Alba García Hernández \\ Centro de Estudios de Consumo \\ Universidad de Castilla-La Mancha
}

\begin{abstract}
Resumen: La Audiencia Nacional ha determinado en Sentencia de 24 de noviembre de 2020 que los datos asociados con el dopaje de un deportista han de ser considerados datos de salud $y$, por esta razón, quedan sujetos a un régimen de especial protección. La lucha contra el dopaje es un movimiento con gran transcendencia en el entorno deportivo, por lo que este pronunciamiento en materia de protección de datos resulta de gran relevancia en este contexto. En este trabajo se abordan diversos asuntos afines con esta cuestión, que exitosamente han resuelto los tribunales españoles, como la delimitación del concepto de salud. Además, se ha intentado aproximar, aunque de manera somera, la noción de datos de salud y su amplio alcance.
\end{abstract}

Palabras clave: protección de datos, dopaje, datos de salud, régimen de protección

Title: Health data concerning to an athlete's doping information: Spanish Court rules on their special protection

\footnotetext{
* Trabajo realizado en el marco del Contrato con referencia 2020-COB-9996 financiado con cargo a la ayuda para la realización de proyectos de investigación científica y transferencia de tecnología, de la Junta de Comunidades de Castilla-La Mancha cofinanciadas por el Fondo Europeo de Desarrollo Regional (FEDER) para el Proyecto titulado "Protección de consumidores y riesgo de exclusión social en Castilla-La Mancha" (PCRECLM) con Ref.: SBPLY/19/180501/000333 dirigido por Ángel Carrasco Perera y Ana Isabel Mendoza Losana; en el marco del Proyecto de Investigación PGC2018-098683-B-I00, del Ministerio de Ciencia, Innovación y Universidades (MCIU) y la Agencia Estatal de Investigación (AEI) cofinanciado por el Fondo Europeo de Desarrollo Regional (FEDER) titulado "Protección de consumidores y riesgo de exclusión social", dirigido por Ángel Carrasco Perera y Encarna Cordero Lobato; a la Ayuda para la financiación de actividades de investigación dirigidas a grupos de la UCLM Ref.: 2020-GRIN-29156, denominado "Grupo de Investigación del Profesor Ángel Carrasco" (GIPAC) y en el marco del Proyecto Convenio de colaboración entre la UCLM y el Ilustre Colegio Notarial De Castilla-La Mancha (17 enero 2014) (OBSV) con referencia CONV140025, que dirige el Prof. Ángel Carrasco Perera.
} 
Revista CESCO, N. ${ }^{\circ} 37 / 2021$

doi.org/10.18239/RCDC_2021.37.2674
Alba García Hernández

Los datos de salud relativos a la información sobre dopaje de un deportista: La Audiencia Nacional se pronuncia sobre su especial protección

Abstract: Data referred to an athlete's doping information must be considered health data and, for that reason, are subject to a reinforced legal protection system according to Spanish Court. This paper addresses several issues related to this affair that has been successfully asked by Spanish Courts, such as the definition of health. Furthermore, an attempt has been made to approximate, albeit in a cursory manner, the notion of health data and its broad scope.

Keywords: data protection, doping, health data, legal protection system

SUMARIO: 1. Introducción. 2. Antecedentes. 3. ¿Qué son datos de salud? 4. El tratamiento de los datos de salud: ¿es posible su publicación? 5. Teniendo en cuenta lo anterior, ¿se puede considerar la información relativa al dopaje de un deportista como dato de salud? 6. ¿Cómo han regulado esta cuestión el resto de los Estados miembros? 7. Conclusiones. 8. Bibliografía.

\section{Introducción}

Con motivo del pronunciamiento reciente de la Audiencia Nacional (en adelante, AN) a favor de la Agencia Española de Protección de Datos (en adelante, AEPD) y de un deportista español se aclara una antigua coyuntura: ¿es la información sobre dopaje un dato de salud del atleta? Tomando en consideración esta cuestión principal, surgen otros asuntos que resolver de manera paralela:

- ¿Qué es salud?

- ¿Qué tipo de información ha de englobarse bajo el concepto de dato de salud?

- ¿Se debe reconocer a los datos de salud un régimen de protección de grado superior ante una potencial injerencia que pudiera afectar a la esfera más privada del paciente?

- ¿Cómo ha sido tratada esta problemática en el marco europeo?

En el presente trabajo se ha pretendido abordar cada uno de estos asuntos en observancia del pronunciamiento emitido por los órganos jurisdiccionales españoles.

\section{Antecedentes}

Con fecha 19 de julio de 2018, la AEPD emitía resolución por la que concluía que la Agencia Española de Protección de la Salud en el Deporte (AEPSAD) había cometido una infracción muy grave por incumplimiento de lo dispuesto en el apartado 3 del art. 7 de la Ley Orgánica 15/1999, de 13 de diciembre, de Protección de Datos de Carácter Personal (en adelante LOPD), en el que se establece que "los datos de carácter personal que hagan referencia al origen racial, a la salud y a la vida sexual sólo podrán ser recabados, tratados y cedidos cuando, por razones de interés general, así lo disponga una ley o el afectado consienta expresamente".

La resolución tiene su origen en la revelación de un dato de salud por parte de la AEPSAD como consecuencia de la incompleta anonimización de un expediente sancionador por dopaje a un deportista. El afectado denunció ante la AEPD que la 
Revista CESCO, N. ${ }^{\circ} 37 / 2021$

doi.org/10.18239/RCDC_2021.37.2674
Alba García Hernández

Los datos de salud relativos a la información sobre dopaje de un deportista: La Audiencia Nacional se pronuncia sobre su especial protección

AEPSAD había revelado datos relativos a su salud al hacer públicas las alegaciones que este había realizado a partir de la apertura de un expediente sancionador por dopaje, en las que manifestó que la presencia en sus pruebas fisiológicas de una sustancia prohibida se debía a la "ingesta accidental de un medicamento que estaba tomando su hijo por una enfermedad común" ${ }^{1}$. Con todo, el denunciante alegaba que la resolución del Tribunal Administrativo del Deporte (en adelante, TAD), en la que se recogían las aclaraciones que él había realizado a este respecto suponía, por parte de la AEPSAD, una revelación de un dato de salud.

La AEPSAD reconoció la publicación del nombre y apellidos del afectado y la circunstancia motivante de la sanción; sin embargo, consideraba que tales factores eran insuficientes para catalogar la infracción como muy grave, al entender que la publicación de la resolución no había referido datos de salud. En consecuencia, esta entidad, al mostrarse en desacuerdo con lo resuelto por la AEPD, recurrió ante la AN.

Por consiguiente, el objeto de la litis es la determinación de si la información sobre dopaje de un deportista ha de entenderse como dato de salud. Así, de acuerdo con tal calificación, la infracción adquirirá una graduación diferente:

- Si se determina que la información relativa al consumo de sustancias prohibidas por parte de deportistas es dato de salud, la infracción se ajustará a lo previsto en el art. 44.4.b) de la LOPD, entonces será tipificada como muy grave.

- Si, por el contrario, el contenido sobre dopaje no se encuentra bajo el alcance de los datos de salud, será de aplicación lo previsto en el art.44.3. g) de la LOPD y la infracción será grave, pues se habría infringido el deber de guardar secreto sobe información de naturaleza personal incorporada en ficheros correspondientes a la comisión de infracciones administrativas.

Es decir, no se discute la existencia de infracción en relación con el tratamiento de los datos personales del deportista sino la calificación que se les otorga.

\section{3. ¿Qué son datos de salud?}

Aportar una definición taxativa de lo que ha de entenderse por salud resulta sumamente complicado, ya que este concepto incluye una amplia diversidad de factores de naturaleza tanto objetiva como subjetiva que condicionan su delimitación conceptual. La Real Academia Española de la Lengua aporta distintas acepciones a este concepto: 1) estado en el que el ser orgánico ejerce normalmente todas sus funciones, 2) conjunto de las condiciones físicas en que se encuentra un organismo en un momento determinado, 3) libertad o bien público o particular de cada uno [...]. Por su parte, la Organización Mundial de la Salud define la salud como un estado de completo bienestar físico, mental y social, y no solamente la ausencia de afecciones o enfermedades.

${ }^{1}$ Vid. FJ $2^{\circ}$ de la SAN (Sala de lo Contencioso) de 24 de noviembre de 2020 (REC 791/2018). 
Revista CESCO, N. ${ }^{\circ} 37 / 2021$

doi.org/10.18239/RCDC_2021.37.2674
Alba García Hernández

Los datos de salud relativos a la información sobre dopaje de un deportista: La Audiencia Nacional se pronuncia sobre su especial protección

Como puede apreciarse, estas definiciones se caracterizan por su amplio alcance, así como por una gran falta de concreción, por lo que, en la misma medida en que es espinosa la tarea de definir el término salud, resulta complejo determinar cuándo nos encontramos ante un dato de salud. No obstante, tanto el legislador comunitario como el nacional son plenamente conscientes de que esta información es susceptible de ser considerada como datos de carácter personal. Es más, reconocen que no todos los datos personales referidos a un individuo lo afectan en la misma medida, sino que algunos de ellos entrañan circunstancias de mayor sensibilidad o vulnerabilidad, incidiendo de forma notable sobre el acervo más íntimo de la persona, como sucede con la información referida a la salud de un sujeto. Es por esta razón que, tanto la doctrina como la jurisprudencia, lo han calificado como dato sensible 2 .

El Real Decreto 1720/2007, de 21 de diciembre, por el que se aprueba el Reglamento de desarrollo de la Ley Orgánica 15/1999, de 13 de diciembre, de protección de datos de carácter personal define los datos de carácter personal relacionados con la salud como aquellas "informaciones concernientes a la salud pasada, presente y futura, física o mental, de un individuo $[\ldots]^{3 \prime}$. Por su parte, la Ley 41/2002, de 14 de noviembre, básica reguladora de la autonomía del paciente y de derechos y obligaciones en materia de información y documentación clínica define la información clínica como "todo dato, cualquiera que sea su forma, clase o tipo, que permite adquirir o ampliar conocimientos sobre el estado físico y la salud de una persona, $o$ la forma de preservarla, cuidarla, mejorarla o recuperarla". Por consiguiente, no solamente se debe entender por dato de salud aquel referido a una enfermedad o disfunción, sino que también lo será toda aquella información que refleje la ausencia de ella, es decir, un buen estado de salud ${ }^{4}$.

A estas primeras aproximaciones se debe añadir las consideraciones que realiza el Reglamento 2019/679 de 27 de abril de 2016 relativo a la protección de datos personales de las personas físicas (en adelante, RGPD) en el que califica como dato de salud tanto aquella información tendente a la identificación del sujeto afectado (nombre, apellidos, datos de contacto, etc.) como al contenido referido al estado de salud de la persona en sentido estricto. Así, incluye:

- Datos vinculados con el estado de salud de la persona, ya fuera pasado, presente o futuro, esto es, cualquier información vinculada con una enfermedad o discapacidad actual o sufrida en el pasado, así como la referente a los potenciales riesgos de padecerla en el futuro. En este punto, se han de considerar incluidas las informaciones concernientes a individuos sanos, enfermos o, incluso, ya fallecidos.

\footnotetext{
2 ABERASTURI GORRIÑO, U., Los Principios de la Protección de Datos Aplicados en la Sanidad, Servicio Editorial de la Universidad del País Vasco, 2011, p. 144.

${ }^{3}$ Art. 5.

${ }^{4}$ Lorenzo Abogados, "Guía Básica para el Cumplimiento de la Ley Orgánica de Protección de Datos en el Sector Sanitario", p.6, disponible en: https://bit.ly/3p19MkU.
} 
Revista CESCO, N. ${ }^{\circ} 37 / 2021$

doi.org/10.18239/RCDC_2021.37.2674
Alba García Hernández

Los datos de salud relativos a la información sobre dopaje de un deportista: La Audiencia Nacional se pronuncia sobre su especial protección

- El conjunto de números, símbolos o cualquier otro dato asignado a un paciente con la finalidad de identificarlo a efectos sanitarios.

- Son datos de salud los contenidos en el historial médico, el tratamiento clínico y los estados biomédicos o fisiológicos de un sujeto.

- La información que hubiera sido recabada a partir de pruebas, exámenes y muestras médicos, genéticos o biológicos.

- Los datos recopilados con motivo de la inscripción a efectos de asistencia sanitaria.

Además, el apartado 45 de la Memoria Explicativa del Convenio 108 del Consejo de Europa hace extensiva la definición de datos de la salud a las informaciones relativas al abuso de alcohol o al consumo de drogas. Igualmente, adquieren tal denominación los datos atinentes a la salud mental del individuo, ya fuera obtenidos directamente a partir de aquellos expedientes médicos originados con motivo de la asistencia y tratamiento médico del paciente o bien, aquella información manifestada por el propio paciente. Esto es así porque el conocimiento de determinados aspectos psicológicos de la persona podría conducir a la identificación de otros rasgos o aspectos de la personalidad del individuo como sus creencias, ideología, etc. ${ }^{5}$.

Bien es cierto que, aunque, en términos generales, se habla de datos de salud y es este el término que se empleará a lo largo del presente trabajo, dentro de esta definición quedan circunscritos diversos conceptos que podrían ser confundidos entre sí: datos médicos, datos de salud y datos sanitarios. En primer lugar, la primera de las distinciones parte del ámbito en el que el dato haya sido recabado y tratado; así, cabría diferenciar entre datos sanitarios cuando se alude a información concerniente a la salud de un individuo tratada en el campo sanitario de aquella información tratada en cualquier otro ámbito distinto a los propios centros e instituciones sanitarias, ya fueran públicas o privadas (ej. Información sobre el estado de salud de un empleado facilitada al empresario en el momento de la contratación). De este modo, se observa que, de forma general, el contenido tratado podría llegar a ser el mismo; sin embargo, la finalidad, el responsable del tratamiento y el sector en el que trata la información son diferentes. De otra parte, se habla de datos médicos cuando se hace referencia exclusiva a la información atinente al estado de salud de una persona -tanto física como mental-, siendo el alcance de esta definición más restringido que el presumible de los datos relativos a la salud, donde no solamente se incluyen los datos médicos, sino aquellos que tienen una vinculación directa con la salud como los hábitos de vida del paciente ${ }^{6}$.

Toda esta información, independientemente de la fuente de la que proceda, tendrá la consideración de datos de salud y, es por ello, que estos datos se encuentran blindados por una especial protección que el propio ordenamiento concede en base a su alto grado de sensibilidad. Este particular reconocimiento que le concede el

\footnotetext{
${ }^{5}$ FERNÁNDEZ LÓPEZ, J. M., El Derecho Fundamental a la Protección de los Datos Personales. Obligaciones que Derivan para el Personal Sanitario, Revista Derecho y salud, 11(1), 37-46, 2003.

${ }^{6}$ ABERASTURI GORRIÑO, U., Los Principios de la Protección de ... op. cit. p. 136-143.
} 
Revista CESCO, N. ${ }^{\circ} 37 / 2021$

doi.org/10.18239/RCDC_2021.37.2674
Alba García Hernández

Los datos de salud relativos a la información sobre dopaje de un deportista: La Audiencia Nacional se pronuncia sobre su especial protección

legislador deriva de la idea de que la salud del paciente es un concepto intrínsecamente ligado a la intimidad de las personas, pues pertenece a su esfera más personal. Es por este motivo que el ordenamiento europeo limita el tratamiento de este tipo de información. En esta línea se ha manifestado TRONCOSO REIGADA, quien ha optado por ligar el derecho a la intimidad, la salud y la vida, exponiendo que "los datos de salud y la historia clínica tienen una enorme importancia porque son instrumentos necesarios para garantizar la asistencia sanitaria de las personas, y por tanto están íntimamente vinculados al derecho a la vida -Art. 15 CE- y a la protección de la salud -Art. 43 CE-. La acumulación de datos sanitarios de los pacientes en las historias clínicas y su correcta conservación son elementos necesarios para poder llevar un seguimiento del estado de salud de las personas. De hecho, la supresión de datos relevantes de la historia clínica puede llegar a afectar gravemente a los tratamientos sanitarios y, en última instancia, a la vida de las personas ${ }^{7 "}$. No obstante, se ha de matizar esta idea, pues el despliegue del conjunto de medidas tendentes a garantizar la salvaguarda de los datos de un individuo, particularmente, aquellos referentes a su salud tendrá lugar cuando la información identifique o haga identificable al sujeto, es decir, no solamente se incluye aquel contenido que individualiza a una persona de forma directa, sino que pudiera permitir una ulterior identificación ${ }^{8}$.

Como ya se mencionaba anteriormente, los datos relativos a la salud son datos sensibles. Tal calificación brinda un régimen "especial" de protección a esta información, aunque la salvaguarda no es suprema, pues se han de distinguir dos niveles de defensa:

- Nivel 1: protección máxima para datos relativos a las creencias, ideología y afiliación sindical (información hipersensible)

- Nivel 2: protección especial para datos vinculados con la salud, la sexualidad y el origen racial (información sensible).

No obstante, la fijación de diversos estratos o niveles de protección de acuerdo con la naturaleza de los datos resulta, en términos pragmáticos, excesivamente complejo ${ }^{9}$. Así, algunas corrientes doctrinales apuestan por ligar la necesidad de protección con el uso que se fuera a hacer de los datos, en detrimento de las posturas tradicionales que abogan por otorgar una protección cualificada en base a la naturaleza de la información tratada ${ }^{10}$.

Resulta imprescindible hacer alusión a que toda esta información será recopilada, presumiblemente, en un mismo cuerpo: la historia clínica del paciente. En ella se recogerán los datos relativos al paciente referidos al tratamiento y asistencia médica

\footnotetext{
7 TROncoso ReIGAdA, A., La protección de Datos Personales: En Busca del Equilibrio, Tirant lo Blanch, Valencia, 2010, p. 1099.

8 TIMÓN HERRERO, M., Protección de Datos de Carácter Personal y Crisis Sanitaria (COVID 19), elderecho.com, 7 de agosto de 2020, disponible en: https://bit.ly/3tHgHTO.

${ }_{9}^{9}$ ABERASTURI GORRIÑO, U., Los Principios de la Protección de ... op. cit. p. 146.

${ }^{10}$ ABERASTURI GORRIÑO, U., Los Principios de la Protección de ... op. cit. p. 147.
} 
Revista CESCO, N. $37 / 2021$

doi.org/10.18239/RCDC_2021.37.2674
Alba García Hernández

Los datos de salud relativos a la información sobre dopaje de un deportista: La Audiencia Nacional se pronuncia sobre su especial protección

que reciba en cualquier momento, así como todas aquellas pruebas médicas que le sean practicadas (análisis de sangre, pruebas gráficas, etc.) ${ }^{11}$. La Ley $41 / 2002$, de 14 de noviembre, básica reguladora de la autonomía del paciente y de derechos y obligaciones en materia de información y documentación clínica define la historia clínica como "el conjunto de documentos que contienen los datos, valoraciones e informaciones de cualquier índole sobre la situación y la evolución clínica de un paciente a lo largo del proceso asistencial"12; por tanto, se presenta como un elemento esencial para los profesionales sanitarios para prestar una asistencia óptima al paciente. Además, es la vía principal por la que toda la información relativa a la salud de un individuo es accesible y centralizada ${ }^{13}$.

Sin embargo, la accesibilidad a esta categoría especial de datos puede entrañar graves daños para la persona afectada por tal "intromisión", por lo que el acceso es limitado y únicamente se posibilita para satisfacer motivos asistenciales, de investigación, docencia epidemiología, de salud pública, judiciales, planificación o evaluación. A pesar de reconocerse la legitimidad de estas finalidades para justificar el acceso a la historia clínica, la que fundamenta cualquier acceso es la prestación de asistencia sanitaria, siendo la más común en el mundo real.

De este modo, podría decirse que la historia clínica se encuentra conectada necesariamente con el derecho de todo paciente a recibir una adecuada asistencia y, en consecuencia, es obligación del personal médico acceder a ella para garantizar la idoneidad del servicio prestado -actualmente se habla de un nuevo principio en el ámbito sanitario y es la vinculación asistencial, que ha de considerarse en concordancia con el principio de minimización de datos, esto es, el facultativa únicamente conocerá de aquellas informaciones que sean requeridas para la correcta asistencia y tratamiento del interesado. De esta forma se dibujan los límites del acceso a la historia clínica-.

A estos derechos y obligaciones se ha de sumar el deber de confidencialidad como complementario del deber de secreto profesional, el cual alcanza a cualquier individuo que tenga acceso a los datos de salud de un paciente con independencia de la profesión que ejerza ${ }^{14}$. La protección de datos afecta en esta materia al conjunto de profesionales que operan en el sector sanitario, ya fuera de manera individual o por medio de hospitales, clínicas o instituciones sanitarias ${ }^{15}$. En este punto, puede decirse que no existe un derecho absoluto de confidencialidad reconocido al paciente, pues, en muchas ocasiones, la realidad práctica obliga a los facultativos a "revelar" esta

\footnotetext{
11 HIDAlgo CEREZO, A., Protección de Datos de Carácter Personal Relativos a la Salud del Paciente: Fundamentos, Protección a la Intimidad y Comentarios al Nuevo Reglamento UE 2016/679, Revista de derecho UNED, 19, 715-744, 2016.

12 Art. 3.

13 CASANOVA ASENCIO, A.S., Protección de Datos en el Ámbito de la Historia Clínica: el Acceso Indebido por el Personal Sanitario y sus Consecuencias, 2019.

${ }^{14}$ CASANOVA ASENCIO, A.S., Protección de Datos en el... op.cit. passim.

15 Lorenzo Abogados, "Guía Básica para el Cumplimiento... op. cit. p.7, disponible en: https://bit.ly/3p19MkU.
} 
Revista CESCO, N. ${ }^{\circ} 37 / 2021$

doi.org/10.18239/RCDC_2021.37.2674
Alba García Hernández

Los datos de salud relativos a la información sobre dopaje de un deportista: La Audiencia Nacional se pronuncia sobre su especial protección

información; sin embargo, sí que podría haberse de confidencialidad absoluta en el ámbito de la relación médico-paciente ${ }^{16}$.

\section{El tratamiento de los datos de salud: ¿es posible su publicación?}

Como se ha venido diciendo, los datos relativos a la salud tienen la consideración de datos sensibles y es por esta razón que el responsable de su tratamiento deberá tener especial cuidado en el desarrollo de sus funciones.

EI RGPD prevé en su art. 9 la necesidad de recabar el consentimiento del afectado de forma explícita y por escrito, salvo que estos vayan a ser utilizados con fines de medicina preventiva o laboral, diagnóstico, tratamiento sanitario o social o para la gestion de los servicios de asistencia sanitaria, de conformidad con los arts. 6.1.b) y c) del RGPD. Tampoco será necesario el consentimiento cuando se requieran los datos médicos por motivos de salud pública ${ }^{17}$.

Asimismo, los datos recopilados del paciente deberán ajustarse a los principios de veracidad, pertinencia, adecuación, licitud e información al paciente. De conformidad con este último principio, los sujetos afectados deberán ser informados en todo momento de la finalidad del tratamiento, de la existencia de ficheros, de los derechos que se le reconocen y de la identidad del responsable del tratamiento. Aquí se ha de distinguir entre la información asistencial y el consentimiento informado ${ }^{18}$ como manifestación consciente y libre del paciente para que tenga lugar una actuación que afecte a su salud ${ }^{19}$. Nuestro Tribunal Supremo se refiere al consentimiento informado como "una de las últimas aportaciones realizadas en la teoría de los derechos humanos, consecuencia necesaria o explicación de los clásicos derechos a la vida, a la integridad física y a la libertad de conciencia"20. MÉNDEZ BAIGES lo define como el acto a través del cual el interesado, en este caso el paciente, autoriza al personal sanitario a practicarle un tratamiento, después de que se le hayan comunicado y explicado debidamente las características de este, por tanto, requiere siempre información terapéutica ${ }^{21}$.

Este derecho a ser informado ha de ser complementado con el derecho a la confidencialidad de los datos, incluso, cuando la relación médico-paciente hubiera finalizado. En este punto, cualquier actividad de tratamiento debe llevar aparejada ciertas medidas de seguridad articuladas a partir del riesgo que entrañe la actividad que, en este caso, se demuestra muy elevado.

Igualmente, como consecuencia de la vulnerabilidad de la información tratada, se habrá de dar preferencia al principio de minimización de datos y de limitación de la

\footnotetext{
${ }^{16}$ CRISTEA UIVARU, L., La Protección de Datos de Carácter sensible: Historia Clínica Digital y Big Data en Salud, La protección de datos de carácter sensible, 1-358, 2018, p. 113.

17 Art. 6.1.e) RGPD.

18 CRISTEA UIVARU, L., La Protección de Datos de Carácter sensible... op. cit, p. 85.

${ }^{19}$ CRISTEA UIVARU, L., La Protección de Datos de Carácter sensible... op. cit, p. 91.

${ }^{20}$ CRISTEA UIVARU, L., La Protección de Datos de Carácter sensible... op. cit, pp. 94-95.

${ }^{21}$ CRISTEA UIVARU, L., La Protección de Datos de Carácter sensible... op. cit, p. 95.
} 
Revista CESCO, N. ${ }^{\circ} 37 / 2021$

doi.org/10.18239/RCDC_2021.37.2674
Alba García Hernández

Los datos de salud relativos a la información sobre dopaje de un deportista: La Audiencia Nacional se pronuncia sobre su especial protección

finalidad. Respecto al primero, es esencial que únicamente sean tratados los datos pertinentes para la satisfacción de los fines establecidos previamente, esto es, aquellos exclusivamente necesarios para prestar la asistencia médica. Por otro lado, los datos empleados se recopilarán de acuerdo con unos fines determinados y lícitos, de forma que no podrán utilizarse para otras finalidades "incompatibles" con las fijadas en el inicio. En este sentido, si se superaran los límites descritos y se actuara de manera no diligente, se entenderá que el consentimiento prestado por el paciente no es eficaz, ya que el tercero autorizado para el tratamiento de los datos habría superado el alcance de las actividades circunscritas bajo el alcance del consentimiento del usuario. En esta línea se ha pronunciado el Tribunal Constitucional manifestando que el consentimiento no desplegará efectos cuando "se subviertan los términos y el alcance para el que se otorgó el consentimiento, quebrando la conexión entre la información personal que se recaba y el objetivo tolerado para el que fue recogida"22.

En cuanto al periodo de conservación de los datos, se fija un plazo de, al menos, cinco años desde que tuviera lugar cada proceso asistencial. Continuando con los plazos previstos en esta materia, cuando concurriera daño alguno contra el interesado como resultado del tratamiento de sus datos de salud, el Código Civil determina un plazo de quince años para el ejercicio de una acción por responsabilidad civil, que comenzará desde el momento en el que el afectado tuviera conocimiento de ello.

De otra parte, a pesar del alto riesgo que supone el tratamiento de esta información sensible, son numerosos los agentes que pueden entrar en contacto con ella durante la asistencia y tratamiento al paciente. De este modo, el legislador determina que únicamente estará facultado a acceder a los datos médicos el personal implicado en la atención de la persona afectada. Por tanto, la libertad de acceso a los datos de salud es plena para el personal sanitario que lo tratara -siempre que la finalidad de acceso fuera legítima y respondiera a intereses asistenciales- y al propio paciente, no así a sus familiares, quienes deberán acreditar un interés legítimo para la puesta en conocimiento de tal información, siempre que el paciente no hubiera manifestado una voluntad contraria.

No solamente se pueden tratar los datos con motivos asistenciales, a pesar de que este sea la finalidad más frecuente de esta actividad, sino que también es posible hacerlo con fines de investigación. Para ello, se deberá recabar el consentimiento expreso del paciente. Si este no se obtuviera, podría llevarse a cabo un proceso de anonimización de los datos identificativos del afectado y así evitar cualquier tipo de identificación ${ }^{23}$. No obstante, en muchas ocasiones, no solo se emplean datos, entendiéndose estos como mera información, sino que se hace uso de fotografías u

22 BERROCAL LANZAROT A.I., La Protección de Datos Relativos a la Salud y la Historia Clínica En la Normativa Española y Europea, Revista de la Escuela de Medicina Legal, (18) 11-44, 2011, p. 28.

23 Agencia Española de Protección de Datos, "Guía para Pacientes y Usuarios de la Sanidad", 2019, disponible en: https://bit.ly/3q3q7Hh. 
Revista CESCO, N. $37 / 2021$

doi.org/10.18239/RCDC_2021.37.2674
Alba García Hernández

Los datos de salud relativos a la información sobre dopaje de un deportista: La Audiencia Nacional se pronuncia sobre su especial protección

otras herramientas audiovisuales que podrían desembocar en la identificación del paciente, por lo que la fórmula más frecuentemente empleada es la de tapar las facciones que podrían hacer identificable al sujeto, aunque esto puede resultar insuficiente. En estos casos, conviene obtener el consentimiento del paciente ${ }^{24}$.

Por otro lado, especialmente controvertida es la comunicación de esta categoría de datos a terceros. Cuando se requiera tal comunicación, necesariamente se deberá informar al paciente y solicitar su consentimiento, salvo que nos encontremos ante alguna de las excepciones recogidas exclusivamente en el ordenamiento de conformidad con el principio de reserva de ley (ej. Cuando la ley prevea expresamente la cesión de datos sin necesidad de recabar el consentimiento del interesado, cuando sea necesario como salvaguarda de la salud pública o cuando se ponga en peligro la integridad psíquica o física del paciente). En todo caso, la comunicación deberá circunscribirse a los fines legítimos que fueron autorizados por el afectado. Así, respecto a la cesión de datos, cabe diferenciar entre la cesión de datos en sentido estricto y el acceso de terceros a los datos de salud. En este último supuesto, se deberá concretar cuál es la relación que vincula a las partes involucradas, así como delimitar las actividades que el tercero podrá desarrollar conforme a las instrucciones que reciba del responsable del tratamiento. Si se incumpliera este régimen, se estaría incurriendo en una infracción de carácter muy grave.

\section{Teniendo en cuenta lo anterior, ¿se puede considerar la información relativa al dopaje de un deportista como dato de salud?}

Pues bien, una vez que se ha tratado de delimitar el concepto de dato de salud, a pesar de la dificultad que comporta esta tarea, conviene avanzar en la siguiente cuestión: ¿se puede considerar la información relativa al dopaje de un deportista como dato de salud? Y, si la respuesta fuera afirmativa, cabría dar respuesta a otra pregunta que se podría plantear a colación de la primera ¿es predicable, en consecuencia, una especial protección de esta tipología de datos?

La Agencia Mundial Antidopaje (AMA) define el dopaje a partir de cuatro circunstancias: "1) presencia de una sustancia prohibida, o de sus metabolitos o marcadores, en la muestra biológica de un deportista; 2) uso, o tentativa de uso, de una sustancia o método prohibido; 3) negarse a pasar un control antidopaje o eludirlo de cualquier manera, sin una justificación válida e 4) incumplimiento de la localización o paradero del deportista". En este contexto, se ha de tener en cuenta que el deportista es el principal responsable de su salud, por lo que, cualquier acción que perjudique o dañe su estado mediante el consumo de una sustancia o el empleo de un método prohibido en el ámbito deportivo, ya fuera de forma premeditada o

\footnotetext{
24 Lorenzo Abogados, "Guía Básica para el Cumplimiento... op. cit. p.19, disponible en:
} https://bit.ly/3p19MkU. 
Revista CESCO, N. ${ }^{\circ} 37 / 2021$

doi.org/10.18239/RCDC_2021.37.2674
Alba García Hernández

Los datos de salud relativos a la información sobre dopaje de un deportista: La Audiencia Nacional se pronuncia sobre su especial protección

inconsciente, supondrá la concurrencia de una situación de dopaje y, por consiguiente, será sancionado en consecuencia ${ }^{25}$.

La lucha contra el dopaje no es un asunto novedoso, sino que, desde hace años, ha supuesto una cuestión controvertida con gran repercusión en la sociedad por sus implicaciones políticas, sociales, médicas y éticas. El Comité Olímpico Internacional construye este movimiento en torno a tres pilares: la integridad del deporte, la protección de la salud y el juego limpio. Tradicionalmente, el argumento más repetido por los contrarios al empleo de prácticas prohibidas en el deporte es la protección que se ha de brindar a los deportistas, pudiéndose entender esta desde dos ópticas distintas:

- desde un punto de vista individual, frente a las potenciales amenazas que pudieran poner en riesgo la integridad física del propio deportista.

- en términos colectivos, se ha de actuar ante las posibles coacciones que pudieran afectar a terceros, es decir, se trata de evitar que el deportista que recurre al dopaje para logar un mejor rendimiento en su actividad coaccione a sus compañeros para que también lo hagan.

Igualmente, la lucha contra el dopaje se erige como muestra innegable de la defensa de los principios del deporte como el esfuerzo y el desafío presumible de las competiciones deportivas, la emoción de la competición y el trabajo en equipo ${ }^{26}$.

En esta línea se pronuncia la AN, quien manifiesta que "la lucha contra el dopaje está estrechamente vinculada con la salud de los deportistas, como no podía ser de otra manera, ya que el uso y consumo de determinados productos pueden falsear el rendimiento individual $y$, por ello, están prohibidos en las competiciones deportivas, pero, además afectan directamente a las condiciones físicas de los usuarios y, por tanto, también su salud". Así, la sentencia recuerda que el concepto de dato de salud incluye los resultados obtenidos a partir de la recogida de muestras corporales con objeto de análisis médicos, a pesar de las alegaciones presentadas por la AEPSAD, quien defiende que "la toma de muestras de deportistas a efectos del control antidopaje no constituye datos de salud"27.

Asimismo, respecto al régimen de protección, en la demanda, la actora remite a otras sentencias emitidas por la AN en las que esta sostenía la idea de que la defensa reforzada que se reconoce a los datos de salud únicamente tiene razón en el ámbito de la medicina, pero no fuera de ese campo. Ante esto, la AN niega tal consideración y entiende que "una cosa es que en la casuística de las resoluciones administrativas de la AEPD y en las sentencias de esta Sala los casos más frecuentes se produzcan en ese ámbito de los servicios sanitarios públicos o privados y otro muy distinto es que fuera de él no tengan la consideración de datos de salud, ya que lo son con

\footnotetext{
25 Agencia Española de Protección de la Salud en el Deporte, "¿Qué es el dopaje?", disponible en: https://bit.ly/3q3cRIY.

26 PÉREZ TRIVIÑO, J. L., Dopaje, Revista en Cultura de la Legalidad, 183-191, 2015.

27 Vid. FJ 40 de la SAN (Sala de lo Contencioso) de 24 de noviembre de 2020 (REC 791/2018).
} 
Revista CESCO, N. ${ }^{\circ} 37 / 2021$

doi.org/10.18239/RCDC_2021.37.2674
Alba García Hernández

Los datos de salud relativos a la información sobre dopaje de un deportista: La Audiencia Nacional se pronuncia sobre su especial protección

independencia de que su tratamiento se produzca en el ámbito de los servicios de atención sanitaria o en otros de otra clase [...] Constituyen una categoría especial de datos que tiene una protección reforzada en la ley, que considera como muy graves las infracciones relativas a su tratamiento o cesión, salvo en los casos y forma legalmente autorizados ${ }^{28} . "$

En efecto, entiende la AN que "no existe, pues, ningún apoyo para la pretensión de la actora ni en la normativa española, ni en la de la Unión Europea, ni en la internacional, de que los datos sobre dopaje en el deporte no son datos de salud del deportista, sin perjuicio de que en la lucha contra el dopaje se regule detalladamente la determinación de existencia de infracciones y su publicidad para evitar el falseamiento de las competiciones $y$, en definitiva, tratar de que el juego sea limpio, pero no se deriva de tales normas que las infracciones en materia de protección de datos no tengan la calificación de gravedad que corresponde a los categorías especiales de datos que están particularmente protegidos, como son los datos de salud ${ }^{29 \prime \prime}$.

Por tanto, la jurisprudencia confirma la calificación de la información sobre dopaje de un deportista como dato de salud y señala la sensibilidad y vulnerabilidad de tal información, reconociéndole un régimen de protección superior. Naturalmente, en palabras de la AN, la sanción que corresponde en el supuesto analizado ha de ser conforme a la comisión de una infracción de carácter muy grave.

\section{6. ¿Cómo han regulado esta cuestión el resto de los Estados miembros?}

Como se ha venido anunciando, la regulación en materia de protección de datos sanitarios en el ordenamiento español se encuentra fraccionada a nivel nacional y autonómico como consecuencia de la ordenación de nuestro territorio. Sin embargo, los cuerpos regulatorios principales son: la Constitución española; la Ley Orgánica $3 / 2018$, de 5 de diciembre, de Protección de Datos Personales y garantía de los derechos digitales (anteriormente la Ley Orgánica 15/1999, de 13 de diciembre, de Protección de Datos de Carácter Personal) y, de forma más específica, la Ley $41 / 2020$, de 14 de noviembre, ley básica reguladora de la autonomía del paciente y de derechos y obligaciones en materia de información y documentación clínica.

Es en el marco jurídico internacional donde tiene lugar el origen de la protección a los datos de naturaleza personal con el art. 12 de la Declaración Universal de los Derechos del Hombre en el que se garantiza la vida privada e intimidad de las personas frente a cualquier tipo de injerencia por parte de terceros. En la misma línea se manifiestan el Convenio para la Protección de los Derechos Humanos y de las Libertades Fundamentales y el Pacto Internacional de Derechos Civiles y Políticos. En el ámbito europeo, uno de los instrumentos normativos más novedosos en esta

\footnotetext{
28 Vid. FJ $5^{\circ}$ de la SAN (Sala de lo Contencioso) de 24 de noviembre de 2020 (REC 791/2018).

${ }^{29}$ Vid. FJ $5^{\circ}$ de la SAN (Sala de lo Contencioso) de 24 de noviembre de 2020 (REC 791/2018).
} 
Revista CESCO, N. ${ }^{\circ} 37 / 2021$

doi.org/10.18239/RCDC_2021.37.2674
Alba García Hernández

Los datos de salud relativos a la información sobre dopaje de un deportista: La Audiencia Nacional se pronuncia sobre su especial protección

materia es el Convenio sobre Derechos Humanos y la Biomedicina del Consejo de Europa.

Considerando todas estas normas, puede advertirse que la regulación española tiene gran influencia de las normas previstas en el ordenamiento europeo e internacional. En este punto, no solamente se ha de hacer referencia a los datos médicos, sino que el alcance de este concepto ha de entenderse en sentido amplio, esto es, no solo se ha de hacer referencia a los datos sanitarios, sino a toda aquella información vinculada con el estado de salud pasado, presente o futuro de un individuo.

En el ámbito europeo surge actualmente la necesidad de construir un cuerpo regulatorio uniforme y pacífico. Con ello, se ha tratado de satisfacer esta necesidad con la promulgación del Reglamento General de Datos, con el que se incluyen importantes novedades en el campo sanitario: se incluyen como datos de la salud dos nuevas categorías de datos como son los datos biométricos y los genéticos.

Con todo, a pesar de los avances, son muchos los retos que se han de abordar todavía, por ejemplo, la cuestión sobre la seguridad de los datos de la salud, la cual preocupa por el surgimiento de nuevas prácticas como el uso por parte de usuarios y consumidores de aplicaciones tecnológicas para el seguimiento de sus datos de salud como el ejercicio diario practicado, las calorías quemadas, la saturación de oxígeno en nuestra sangre o las horas de sueño. Estos datos de salud son obtenidos por grandes corporaciones a las que se habilita para gestionar y hacer uso de esta información privada. De este modo, ante el acuciante crecimiento del empleo de las nuevas tecnologías, los Gobiernos están comenzando a tomar medidas. Así pues, por ejemplo, la Comisión Federal de Comercio de Estados Unidos ha limitado la cesión de datos médicos por parte de estas grandes empresas a terceros, prohibiendo su comercialización ${ }^{30}$.

En consecuencia, puede decirse que los Estados miembros muestran una gran preocupación respecto a la protección de los datos de carácter personal, particularmente, en relación a aquellos catalogados como especialmente sensibles, tal y como sucede con los datos relativos a la salud, a los que se les reconoce un régimen de protección reforzado. El ordenamiento europeo, en observancia de sus normas, acuerda la consideración de la información sobre dopaje atinente a un deportista como dato de salud; por lo que este contenido puede ser susceptible de circunscribirse a un sistema de salvaguardia fortalecido.

\section{Conclusiones}

Tras este exhaustivo análisis se puede concluir que delimitar qué ha de reconocerse como dato de salud es una complicada tarea, debido a la falta de concreción y a la gran amplitud presumible del término salud. A pesar de la dificultad, el legislador y la jurisprudencia han centrado sus esfuerzos en precisar esta noción, en la que se distingue una especial susceptibilidad, ya que cualquier injerencia no permitida que

\footnotetext{
${ }^{30}$ CRISTEA UIVARU, L., La Protección de Datos de Carácter sensible... op. cit. p. 233.
} 
tenga lugar con el tratamiento de datos médicos supone una grave intromisión en la esfera más íntima y privada de cualquier sujeto.

En consecuencia, se ha apostado por dotar de una protección reforzada a toda aquella información concerniente a la salud, ya fuera información atinente al estado de salud (física o psicológica) o ausencia de esta y a los datos identificativos del paciente. Además, dentro de esta definición se ha de integrar información tan diversa como los datos relativos al consumo de drogas y alcohol o los hábitos de vida del individuo.

Fruto de esta especial sensibilidad que se otorga a los datos vinculados con la salud, la actividad de tratamiento a la que esta información puede ser sometida está restringida al cumplimiento de normas de carácter más estricto, particularmente, en lo que se refiere a la comunicación o cesión de los datos relativos a un paciente a un tercero. En este aspecto, cabe resaltar que el deber de confidencialidad no es absoluto en términos generales, aunque se han de distinguir dos circunstancias: por un lado el deber de confidencialidad que ha de regir la relación paciente-facultativo sí que puede definirse como plena en cuanto al deber de confidencialidad, no así con respecto a terceros, pues, en ocasiones, es necesaria la cesión a agentes externos a esta relación para la correcta prestación del servicio de asistencia sanitaria y su ulterior seguimiento.

De otra parte, estrechamente ligada a la cuestión de la salud se encuentra la problemática del dopaje en el deporte. La lucha contra esta práctica no se revela como cuestión novedosa, sino que ha supuesto una preocupación constante en el entorno deportivo. Como resultado de la conjunción de estos dos asuntos, surge la pregunta sobre la que reza la litis planteada ante la AN: ¿se consideran datos de salud las informaciones vertidas sobre el dopaje de un atleta? A este respecto, la AN sostiene la idea de que los datos de dopaje relativos a un deportista han de ser considerados, igualmente, como datos de salud, pues se refieren directamente al estado de salud del individuo afectado. Este órgano valora que, de manera simultánea a la asimilación de los datos de dopaje con otros datos vinculados con la salud, se ha de conceder un régimen de protección sui generis, basado en la delicadeza y vulnerabilidad de esta información. Esta calificación que han otorgado los órganos españoles la han aplicado igualmente sus homólogos europeos.

\section{Bibliografía}

ABERASTURI GORRIÑO, U., Los Principios de la Protección de Datos Aplicados en la Sanidad, Servicio Editorial de la Universidad del País Vasco, 2011.

Agencia Española de Protección de Datos, "Guía para Pacientes y Usuarios de la Sanidad", 2019, disponible en: https://bit.ly/3q3q7Hh.

Agencia Española de Protección de la Salud en el Deporte, "¿Qué es el dopaje?", disponible en: https://bit.ly/3q3cRIY. 
BERROCAL LANZAROT A.I., La Protección de Datos Relativos a la Salud y la Historia Clínica En la Normativa Española y Europea, Revista de la Escuela de Medicina Legal, (18) 11-44, 2011.

CASANOVA ASENCIO, A.S., Protección de Datos en el Ámbito de la Historia Clínica: el Acceso Indebido por el Personal Sanitario y sus Consecuencias, 2019.

CRISTEA UIVARU, L., La Protección de Datos de Carácter sensible: Historia Clínica Digital y Big Data en Salud, La protección de datos de carácter sensible, 1-358, 2018.

FERNÁNDEZ LÓPEZ, J. M., El Derecho Fundamental a la Protección de los Datos Personales. Obligaciones que Derivan para el Personal Sanitario, Revista Derecho y salud, 11(1), 37-46, 2003.

HIDALGo CEREZO, A., Protección de Datos de Carácter Personal Relativos a la Salud del Paciente: Fundamentos, Protección a la Intimidad y Comentarios al Nuevo Reglamento UE 2016/679, Revista de derecho UNED, 19, 715-744, 2016.

Lorenzo Abogados, "Guía Básica para el Cumplimiento de la Ley Orgánica de Protección de Datos en el Sector Sanitario", disponible en: https://bit.ly/3p19MkU.

PÉREZ TRIVIÑO, J. L., Dopaje, Revista en Cultura de la Legalidad, 183-191, 2015.

TIMÓN HERRERO, M., Protección de Datos de Carácter Personal y Crisis Sanitaria (COVID 19), elderecho.com, 7 de agosto de 2020, disponible en: https://bit.ly/3tHgHTO.

TRONCOSO REIGADA, A., La protección de Datos Personales: En Busca del Equilibrio, Tirant lo Blanch, Valencia, 2010. 